\title{
A vos risques et «perish»
}

\section{Vincent Menuz}

Post-doctorant en bioéthique

Collusion d'intérêt:

Je suis chercheur post-doctoral et donc sujet au publish or perish et au h-index.
Correspondance: Dr Vincent Menuz Université de Montréal Centre de Recherche en Ethique C.P. 6128 Succursale Centre-Ville CA-Montréal (QC) Canada H3C 3J7

vincent.menuz[at]umontreal.ca
Depuis de nombreuses années circule un concept qui se murmure dans les couloirs des universités: celui $\mathrm{du}$ «publish or perish» qui décrit la nécessité pour les chercheurs de publier continuellement - et si possible, rapidement - dans le but d'optimiser leurs chances de réussite dans leur carrière universitaire. Ce n'est pas encore un «principe universitaire» comme l'écrivent certains [1] mais la conséquence directe d'une réalité académique qui choisit la performance, mesurée en nombre de publications, comme critère de sélection de ses chercheurs [2]. Cette réalité peut inciter certains universitaires à publier des articles sans véritables nouveautés ni importance, dans le but unique d'augmenter leur liste de publications. Ils participent ainsi à «l'avalanche» de papiers qui ne sont - et ne seront sans doute - jamais cités [3], ni même lus. C'est ainsi qu'en pressant les chercheurs à la performance sur la base de leur production d'articles, le système académique actuel pousse, bien malgré lui, à la médiocrité.

Une nouvelle façon d'évaluer les chercheurs est en train de se mettre en place dans les universités du monde entier. Un moyen simple, propre, carré, efficace: un moyen numérique. Quoi de plus simple, en effet, que de déterminer la qualité du travail d'un chercheur par un chiffre? Plus ce numéro est élevé, plus le chercheur est considéré comme faisant partie des «meilleurs». Voilà dévoilé l'implacable index de Hirsh, plus connu sous le nom sexy du h-index (ou facteur $\mathrm{H}$ en français) [4]. Cette valeur numérique se calcule non plus seulement sur la liste de publications d'un auteur, mais aussi sur leur taux de cita- tions. Pour faire simple, 12 articles qui sont cités chacun 12 fois donnent un index de 12 , une valeur suffisante pour espérer un poste de professeur associé dans une bonne université en Amérique du Nord [4]. Pour vous donner une idée des fourmis que nous sommes, Albert Einstein et Charles Darwin naviguent respectivement à 96 et 63 . Que les débutants et leur modeste liste de publications se rassurent: il existe, depuis peu, le futur h-index qui permet de prédire leur «potentiel» de publication à cinq ans [5].

\section{Le système académique actuel pousse, bien malgré lui,} à la médiocrité.

C'est quand même bien pensé et quasiment miraculeux: on peut enfin savoir non seulement qui, dans le monde, est le meilleur dans un domaine académique donné, mais aussi celui qui sera «potentiellement» le meilleur dans un quinquennat. Bref, c'est le cauchemar de tous les politiciens. Et peut-être bien aussi celui des chercheurs.

Le h-index et, de manière générale, tous les indicateurs bibliométriques, permettent de mesurer la performance des chercheurs. Ils sont ainsi de plus en plus souvent utilisés à des fins décisionnelles lorsqu'il s'agit de financer le travail d'un chercheur ou de lui attribuer une position académique. Dès lors, ces indicateurs ne se réduisent plus à un simple nombre,
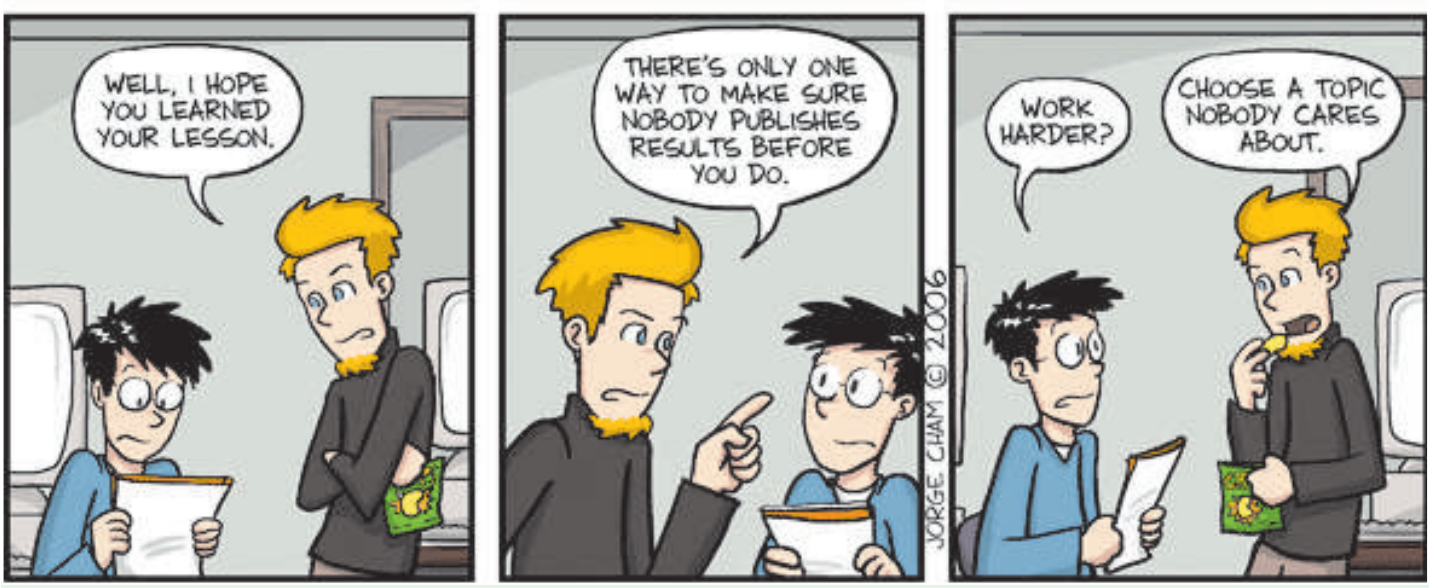

Piled Higher and Deeper by Jorge Cham www.phdcomics.com 
mais deviennent une finalité qui guident le comportement des chercheurs [6], ces derniers devant alors nécessairement œuvrer dans le but d'entrer dans le palmarès des «meilleurs». Le fonctionnement acadé- garanti par son nombre de citations. En attendant qu'un tel dispositif se mette en place, je vais tenter de laisser mes collègues à leur course folle dans leur quête d'être cités. Et cela, à mes risques et perish.

\section{Cette réalité peut inciter certains universitaires à publier des articles sans véritables nouveautés ni importance.}

mique actuel s'apparente ainsi de plus en plus au monde du sport professionnel, comme le suggère un communiqué récent de l'Université de Montréal qui se targue d'employer les «champions» de la physique, de la criminologie et de la communication [1]. Le monde académique verra-t-il bientôt l'émergence d'une culture du dopage, comme c'est le cas dans le cyclisme par exemple [7], avec ses dopants et ses dopés? Ce scénario est loin d'être fantaisiste lorsque l'on sait qu'un certain nombre de chercheurs ont déjà recours à des substances qui leur permettent d'optimiser leurs performances intellectuelles [8].

Pour ma part, je n'écris pas dans le but d'être cité, mais dans celui d'être lu. Et les papiers que je cite dans mes publications ne représentent jamais qu'une infime partie des centaines d'articles que je lis et qui contribuent, d'une manière ou d'une autre, à bâtir ma connaissance et mon expertise sur divers sujets. Et si, comme je le crains, les outils bibliométriques deviennent le passage obligé pour toute carrière académique, je propose alors que ce soit sur la base du nombre de lecteurs - qu'ils appartiennent soit au public à travers la grande presse, ou qu'ils soient spécialisés et se réfèrent à des revues scientifiques - et non sur le nombre de citations que l'impact d'un article soit jugé. En effet, le fait qu'une publication soit citée un grand nombre de fois ne garantit aucunement qu'elle a bénéficié d'un grand lectorat et son impact ne peut donc être logiquement

\section{Références}

1 Sauvé MR. L'UdeM championne en physique, en criminologie et en communication. Forum. 4 septembre 2012. www.nouvelles.umontreal.ca/ recherche/noschercheurs/20120904-ludemchampionne-en-physique-en-criminologie-et-encommunication.html, accédé le 5 octobre 2012)

2 Casadevall A, Fang FC. Reforming Science: Methodological and Cultural Reforms. Infection and Immunity. 2012;80:891-6. (http://iai.asm.org/ content/80/3/891.full, accédé le 5 octobre 2012).

3 Bauerlein M, Gad-el-Hak M, Grody W, McKelvey B, Trimble SW. We must stop the avalanche of low-quality research. The Chronicle of higher education. juin 2010. (http://chronicle.com/article/We-MustStop-the-Avalanche-of/65890/, accédé le 5 octobre 2012).

4 Hirsch JE. An index to quantitify an individual's scientific research output. PNAS. 2005;102:16569-72.

5 Acuna DE, Allesina S, Kording KP. Predicting scientific success. Nature. 2012;489:201-2.

6 Piron S. Lisons Peter Lawrence, ou les implications morales de l'évaluation bibliométrique. Evaluation de la recherche en SHS 6 décembre. 2008. (http:// evaluation.hypotheses.org/229, accédé le 5 octobre 2012).

7 Lentillon-Kaestner V, Brissonneau C. Appropriation progressive de la culture du dopage dans le cyclisme. Déviance et Société . 2009;33:519-41.

8 Sahakian B, Morein-Zamir S. Professor's little helper. Nature. 2007;450:1157-9. 